\title{
Randomized Complexity Lower Bound for Arrangements and Polyhedra*
}

\author{
D. Grigoriev
}

IMR Université de Rennes-1, Beaulieu 35042, Rennes, France

dima@maths.univ-rennes1.fr

\begin{abstract}
The complexity lower bound $\Omega(\log N)$ for randomized computation trees is proved for recognizing an arrangement or a polyhedron with $N$ faces. This provides, in particular, the randomized lower bound $\Omega(n \log n)$ for the DISTINCTNESS problem and generalizes [11] where the randomized lower bound $\Omega\left(n^{2}\right)$ was ascertained for the KNAPSACK problem. The core of the method is an extension of the lower bound from [8] on the multiplicative complexity of a polynomial.
\end{abstract}

\section{Introduction}

The complexity lower bounds for deterministic algebraic computation trees were obtained in [26], [2], [4], [30], [31], [23], and [22] where the topological methods were developed. In particular, these methods provide the lower bound $\Omega(\log N)$ for recognizing (a membership to) a union of planes (of different dimensions) with $N$ faces, by a face we mean any nonempty intersection of several among these planes. As a consequence we obtain the lower bound $\Omega(n \log n)$ for the DISTINCTNESS problem $\bigcup_{1 \leq i<j \leq n}\left\{X_{i}=\right.$ $\left.X_{j}\right\} \subset \mathbb{R}^{n}$ and the EQUALITY SET problem $\left\{\left(x_{1}, \ldots, x_{n}, y_{1}, \ldots, y_{n}\right):\left(x_{1}, \ldots, x_{n}\right)\right.$ is a permutation of $\left.\left(y_{1}, \ldots, y_{n}\right)\right\} \subset \mathbb{R}^{2 n}$, and the lower bound $\Omega\left(n^{2}\right)$ for the KNAPSACK problem $\bigcup_{I \subset\{1, \ldots, n\}}\left\{\sum_{i \in I} x_{i}=1\right\} \subset \mathbb{R}^{n}$. In [14] and [15] a differential-geometric approach for recognizing polyhedra (to which the mentioned topological methods are not applicable) was proposed which gives the lower bound $\Omega(\log N / \log \log N)$, where $N$ is the number of faces of the polyhedron.

\footnotetext{
* This research was supported by NSF Grant CCR-9424358.
} 
The first results on randomized computation trees (RCTs) appeared in [20], [21], [9], and [10] but for a decade an open problem remained: how to obtain nonlinear complexity lower bounds for recognizing natural problems by an RCT. In [12] for the first time the nonlinear lower bound was obtained for a somewhat weaker computational model of randomized algebraic decision trees, in which the testing polynomials in the branching nodes are of a fixed degree, rather than computation trees, in which the testing polynomials are computed along the path of the computation, so they could have in principle an exponential degree. The approach of [12] provides the lower bound $\Omega(\log N)$ for recognizing an arrangement, i.e., a union of hyperplanes, and for recognizing a polyhedron, where $N$ is again the number of faces. In particular, this leads to the lower bound $\Omega(n \log n)$ for the DISTINCTNESS problem and $\Omega\left(n^{2}\right)$ for the KNAPSACK problem. For the EQUALITY SET problem a complexity lower bound on a randomized algebraic decision tree seems to be an open question.

However, the method of [12] does not provide a lower bound for the more interesting model of RCTs. Only in [11] was a method developed which gives, in particular, a lower bound $\Omega\left(n^{2}\right)$ for the KNAPSACK problem on an RCT. This method relies on the lower bound on the multiplicative border complexity of polynomials obtained in [11]. The lower bound $\Omega(\log N)$ of [11] holds for arrangements or polyhedra which satisfy some special conditions which fail, for example, for the DISTINCTNESS problem.

In [8] the proposed lower bound $\Omega(\log N)$ was proved for randomized algebraic computation trees over an arbitrary field of zero characteristic, here the computation branches according to the signs $\{=, \neq\}$ unlike the more customary computation trees over the reals, studied in all previously mentioned papers including this one, which branch according to the signs $\{\leq,>\}$. The core of the method of [8] was the lower bound $\Omega(\log N)$ on the multiplicative complexity of a polynomial (see, e.g., [27]), where $N$ is the number of faces of an arrangement on which the polynomial vanishes.

In the present paper the latter lower bound $\Omega\left(\log N_{1}\right)$ on the multiplicative complexity of a polynomial is extended (see the corollary in Section 2) to a modified invariant $N_{1}$ of an arrangement, namely, the number of so-called strongly singular faces (see Section 1) of the arrangement (now the polynomial does not necessarily vanish on the arrangement). Relying on this lower bound on the multiplicative complexity, the proof of the complexity lower bound $\Omega(\log N)$ for an RCT recognizing an arrangement or a polyhedron with $N$ faces (see the theorem in Section 3) becomes much simpler than the related ones in [12] and [11]. In particular, this gives the lower bound $\Omega(n \log n)$ for an RCT recognizing the DISTINCTNESS problem. The construction of an RCT with linear complexity $O(n)$ for the EQUALITY SET problem from [5] shows that the condition imposed in the present paper (as well as in [8]), that the recognized set is an arrangement, so a union of hyperplanes, rather than a union of planes of greater than 1 codimensions as in the EQUALITY SET problem, is essential. In Section 4 we generalize the construction of [5] and design an RCT for recognizing the following problem $\left\{\left(x_{1}, \ldots, x_{n}, y_{1}, \ldots, y_{m}\right)\right.$ : each of both the differences of the multisets $\left\{x_{1}, \ldots, x_{n}\right\}$ and $\left\{y_{1}, \ldots, y_{m}\right\}$ contains at most $k$ elements $\} \subset \mathbb{R}^{n+m}$ which has a linear complexity when $k$ is a constant. For arbitrary $n, m$ the randomized complexity of this problem remains an open question.

We also mention the paper [13] where a complexity lower bound was established for randomized analytic decision trees (rather than for more customary algebraic ones) and also the paper [7] where a lower bound was ascertained for a randomized parallel 
computational model (rather than a sequential model considered in the quoted papers including the present one).

\section{Strongly Singular Faces of an Arrangement with Respect to a Polynomial}

By $F$ we denote a field of zero characteristic. Let $H_{1}, \ldots, H_{m} \subset F^{n}$ be hyperplanes and let $\Gamma=H_{i_{1}} \cap \cdots \cap H_{i_{n-k}}$ have the dimension $\operatorname{dim} \Gamma=k$, so $\Gamma$ is $k$-face of the arrangement $S=H_{1} \cup \cdots \cup H_{m}$.

Fix arbitrary coordinates $Z_{1}, \ldots, Z_{k}$ in $\Gamma$. Then treating $H_{i_{1}}, \ldots, H_{i_{n-k}}$ as the coordinate hyperplanes of the coordinates $Y_{1}, \ldots, Y_{n-k}$, the coordinates $Z_{1}, \ldots, Z_{k}, Y_{1}, \ldots$, $Y_{n-k}$ in $F^{n}$ are obtained. The next construction of the leading terms of a polynomial is similar to [12] and [11].

For any polynomial $f\left(Z_{1}, \ldots, Z_{k}, Y_{1}, \ldots, Y_{n-k}\right) \in F\left[Z_{1}, \ldots, Z_{k}, Y_{1}, \ldots, Y_{n-k}\right]$, following [12] and [11] define its leading term

$$
\alpha Z_{1}^{m_{1}^{\prime}} \cdots Z_{k}^{m_{k}^{\prime}} Y_{1}^{m_{1}} \cdots Y_{n-k}^{m_{n-k}}
$$

$0 \neq \alpha \in F$, with respect to the coordinate system $Z_{1}, \ldots, Z_{k}, Y_{1}, \ldots, Y_{n-k}$ as the minimal term in the lexicographical ordering $Z_{1}>\cdots>Z_{k}>Y_{1}>\cdots>Y_{n-k}$, namely as follows. First take the minimal integer $m_{n-k}$ such that $Y_{n-k}^{m_{n-k}}$ occurs in the terms of $f=f^{(0)}$. Consider the polynomial

$$
\begin{aligned}
0 \not f^{(1)} & =\left(\frac{f}{Y_{n-k}^{m_{n-k}}}\right)\left(Z_{1}, \ldots, Z_{k}, Y_{1}, \ldots, Y_{n-k-1}, 0\right) \\
& \in F\left[Z_{1}, \ldots, Z_{k}, Y_{1}, \ldots, Y_{n-k-1}\right]
\end{aligned}
$$

which could be viewed as a polynomial on the hyperplane $H_{i_{n-k}}$. Observe that $m_{n-k}$ depends only on $H_{i_{n-k}}$ and not on $Z_{1}, \ldots, Z_{k}, Y_{1}, \ldots, Y_{n-k-1}$, since a linear transformation of the coordinates $Z_{1}, \ldots, Z_{k}, Y_{1}, \ldots, Y_{n-k-1}$ changes the coefficients (being the polynomials from $F\left[Z_{1}, \ldots, Z_{k}, Y_{1}, \ldots, Y_{n-k-1}\right]$ ) of the expansion of $f$ in the variable $Y_{n-k}$, and a coefficient vanishes identically if and only if it vanishes identically after the transformation. Then $f^{(1)}$ is the coefficient of the expansion of $f$ at the power $Y_{n-k}^{m_{n-k}}$.

Second, take the minimal integer $m_{n-k-1}$ such that $Y_{n-k-1}^{m_{n-k-1}}$ occurs in the terms of $f^{(1)}$. In other words, $Y_{n-k-1}^{m_{n-k-1}}$ is the minimal power of $Y_{n-k-1}$ occurring in the terms of $f$ in which the power $Y_{n-k}^{m_{n-k}}$ occurs. Therefore, $m_{n-k}, m_{n-k-1}$ depend only on the hyperplanes $H_{n-k}, H_{n-k-1}$ and not on $Z_{1}, \ldots, Z_{k}, Y_{1}, \ldots, Y_{n-k-2}$, since (as above) a linear transformation of the coordinates $Z_{1}, \ldots, Z_{k}, Y_{1}, \ldots, Y_{n-k-2}$ changes the coefficients (being the polynomials from $F\left[Z_{1}, \ldots, Z_{k}, Y_{1}, \ldots, Y_{n-k-2}\right]$ ) of the expansion of $f$ in the variables $Y_{n-k}, Y_{n-k-1}$ and a coefficient vanishes identically if and only if it vanishes identically after the transformation. Denote by $0 \not \equiv f^{(2)} \in F\left[Z_{1}, \ldots, Z_{k}\right.$, $\left.Y_{1}, \ldots, Y_{n-k-2}\right]$ the coefficient of the expansion of $f$ at the monomial $Y_{n-k-1}^{m_{n-k-1}} Y_{n-k}^{m_{n-k}}$. Obviously

$$
f^{(2)}=\left(\frac{f^{(1)}}{Y_{n-k-1}^{m_{n-k-1}}}\right)\left(Z_{1}, \ldots, Z_{k}, Y_{1}, \ldots, Y_{n-k-2}, 0\right)
$$


One could view $f^{(2)}$ as a polynomial on the $(n-2)$-dimensional plane $H_{i_{n-k}} \cap H_{i_{n-k-1}}$.

Continuing in a similar way, we obtain consecutively the (nonnegative) integers $m_{n-k}$, $m_{n-k-1}, \ldots, m_{1}$ and the polynomials

$$
0 \not \equiv f^{(l)} \in F\left[Z_{1}, \ldots, Z_{k}, Y_{1}, \ldots, Y_{n-k-l}\right],
$$

$1 \leq l \leq n-k$, by induction on $l$. Herewith, $Y_{n-k-l+1}^{m_{n-k-l+1}}$ is the minimal power of $Y_{n-k-l+1}$ occurring in the terms of $f$, in which the monomial $Y_{n-k-l+2}^{m_{n-k-1+2}} \cdots Y_{n-k}^{m_{n-k}}$ for each $1 \leq l \leq n-k$ occurs. Notice that $m_{n-k}, \ldots, m_{n-k-l}$ depend only on the hyperplanes $H_{i_{n-k}}, \ldots, H_{i_{n-k-l}}$ and not on $Z_{1}, \ldots, Z_{k}, Y_{1}, \ldots, Y_{n-k-l-1}$. Then $f^{(l)}$ is the coefficient of the expansion of $f$ at the monomial $Y_{n-k-l+1}^{m_{n-k-l+1}} \cdots Y_{n-k}^{m_{n-k}}$ and

$$
f^{(l+1)}=\left(\frac{f^{(l)}}{Y_{n-k-l}^{m_{n-k-l}}}\right)\left(Z_{1}, \ldots, Z_{k}, Y_{1}, \ldots, Y_{n-k-l-1}, 0\right) .
$$

Thus, $f^{(l)}$ depends only on $H_{i_{n-k}}, \ldots, H_{i_{n-k-l}}$ and not on $Z_{1}, \ldots, Z_{k}, Y_{1}, \ldots, Y_{n-k-l-1}$. One could view $f^{(l)}$ as a polynomial on the $(n-l)$-dimensional plane $H_{i_{n-k}} \cap \cdots \cap H_{i_{n-k-l+1}}$. Continuing, we define also $m_{k}^{\prime}, \ldots, m_{1}^{\prime}$. Observe that the leading term $\operatorname{lm}\left(f^{(l)}\right)=$ $\alpha Z_{1}^{m_{1}^{\prime}} \cdots Z_{k}^{m_{k}^{\prime}} Y_{1}^{m_{1}} \cdots Y_{n-k-l}^{m_{n-k-l}}$, we refer to this equality as the maintenance property (see also [12] and [11]).

From now on the construction and the definitions differ from the ones in [12] and [11]. For any polynomial $g \in F\left[X_{1}, \ldots, X_{n}\right]$ one can rewrite it in the coordinates $\bar{g}\left(Z_{1}\right.$, $\left.\ldots, Z_{k}, Y_{1}, \ldots, Y_{n-k}\right)$ and expand $\bar{g}=g_{s}+g_{s+1}+\cdots+g_{s_{1}}$, where $g_{j} \in F\left[Z_{1}, \ldots, Z_{k}\right.$, $\left.Y_{1}, \ldots, Y_{n-k}\right], s \leq j \leq s_{1}$, is homogeneous with respect to the variables $Y_{1}, \ldots, Y_{n-k}$ of degree $j$ and $g_{s}=g_{s}^{(0)} \not \equiv 0$. Consider the leading term $\operatorname{lm}\left(g_{s}\right)=\alpha Z_{1}^{m_{1}^{\prime}} \cdots Z_{k}^{m_{k}^{\prime}}$ $Y_{1}^{m_{1}} \cdots Y_{n-k}^{m_{n-k}}$ and denote by $\operatorname{Var}^{\left(H_{i_{1}}, \ldots, H_{i_{n-k}}\right)}(g)$ the number of positive (in other words, nonzero) integers among $m_{n-k}, \ldots, m_{1}$, note that $s=m_{1}+\cdots+m_{n-k}$. Although $\operatorname{Var}^{\left(H_{i_{1}}, \ldots, H_{i_{n-k}}\right)}(g)$ depends on the order of the hyperplanes $H_{i_{1}}, \ldots, H_{i_{n-k}}$, we sometimes denote it by $\operatorname{Var}^{(\Gamma)}(g)$ for brevity when no ambiguity could happen. As we have shown above $\operatorname{Var}^{\left(H_{i_{1}}, \ldots, H_{i_{n-k}}\right)}(\mathrm{g})$ is independent from the coordinates $Z_{1}, \ldots, Z_{k}$ of $\Gamma$. Obviously, $\operatorname{Var}^{\left(H_{i_{1}}, \ldots, H_{i_{n-k}}\right)}(g)$ coincides with the number of $1 \leq l \leq n-k$ such that $Y_{n-k-l} \mid g_{s}^{(l)}$, the latter condition is equivalent to that the variety $\left\{g_{s}^{(l)}=0\right\} \cap H_{i_{n-k}} \cap \cdots \cap H_{i_{n-k-l+1}}$ contains the plane $H_{i_{n-k}} \cap \cdots \cap H_{i_{n-k-l+1}} \cap H_{i_{n-k-l}}$ (being a hyperplane in $H_{i_{n-k}} \cap \cdots \cap H_{i_{n-k-l+1}}$ ).

It is convenient (see also [12] and [11]) to reformulate the introduced concepts by means of infinitesimals in case of a real closed field $F$ (see, e.g., [18]). We say that an element $\varepsilon$ transcendental over $F$ is an infinitesimal (relative to $F$ ) if $0<\varepsilon<a$ for any element $0<a \in F$. This uniquely induces the order on the field $F(\varepsilon)$ of rational functions and further on the real closure $\widetilde{F(\varepsilon)}$ (see [18]).

The order in $\widetilde{F(\varepsilon)}$ could be made clearer by embedding it in the larger real closed field $F\left(\left(\varepsilon^{1 / \infty}\right)\right)$ of a Puiseux series (see, e.g., [16]). A nonzero Puiseux series has the form $b=\sum_{i \geq i_{0}} \beta_{i} \varepsilon^{i / \delta}$, where $-\infty<i_{0}<\infty$ is an integer, $\beta_{i} \in F$ for every integer $i ; \beta_{i_{0}} \neq 0$ and the denominator of the rational exponents $\delta \geq 1$ is an integer. The order on $F\left(\left(\varepsilon^{1 / \infty}\right)\right)$ is defined as follows: $\operatorname{sgn}(b)=\operatorname{sgn}\left(\beta_{i_{0}}\right)$. When $i_{0} \geq 1$, then $b$ is called an infinitesimal, when $i_{0} \leq-1$, then $b$ is called infinitely large. For any not infinitely large $b$ we define its standard part $s t(b)=s t_{\varepsilon}(b) \in F$ as follows: when $i_{0}=0$, then $s t(b)=\beta_{i_{0}}$, when $i_{0} \geq 1$, then $s t(b)=0$. In the natural way we extend the standard part to the vectors from $\left(F\left(\left(\varepsilon^{1 / \infty}\right)\right)\right)^{n}$ and further to subsets in this space. 
Now let $\varepsilon_{1}>\varepsilon_{2} \cdots>\varepsilon_{n+1}>0$ be infinitesimals, where $\varepsilon_{1}$ is an infinitesimal relative to $\mathbb{R}$; in general $\varepsilon_{i+1}$ is an infinitesimal relative to $\mathbb{R}\left(\varepsilon_{1}, \ldots, \varepsilon_{i}\right)$ for all $0 \leq i \leq n$. Denote the real closed field $\mathbb{R}_{i}=\mathbb{R}\left(\varepsilon_{1}, \ldots, \varepsilon_{i}\right)$, in particular, $\mathbb{R}_{0}=\mathbb{R}$. For an element $b \in \mathbb{R}_{n+1}$ for brevity denote the standard part $s t_{i}(b)=s t_{\varepsilon_{i+1}}\left(s t_{\varepsilon_{i+2}} \cdots\left(s t_{\varepsilon_{n+1}}(b) \cdots\right)\right) \in \mathbb{R}_{i}$ (provided that it is definable).

We also use Tarski's transfer principle [29]. Namely, for two real closed fields $F_{1} \subset F_{2}$ a closed (so without free variables) formula in the language of the first-order theory of $F_{1}$ is true over $F_{1}$ if and only if this formula is true over $F_{2}$.

An application of Tarski's transfer principle is the concept of the completion. Let $F_{1} \subset F_{2}$ be real closed fields and let $\Psi$ be a formula (with quantifiers and, perhaps, with $n$ free variables) of the language of the first-order theory of the field $F_{1}$. Then $\Psi$ determines a semialgebraic set $V \subset F_{1}^{n}$. The completion $V^{\left(F_{2}\right)} \subset F_{2}^{n}$ is a semialgebraic set determined by the same formula $\Psi$ (obviously, $V \subset V^{\left(F_{2}\right)}$ ).

One could easily see that for any point $\left(z_{1}, \ldots, z_{k}\right) \in \mathbb{R}_{k}^{k}$ and a polynomial $g \in$ $\mathbb{R}\left[X_{1}, \ldots, X_{n}\right]$ such that $g_{s}^{(n-k)}\left(z_{1}, \ldots, z_{k}\right) \neq 0$ (we utilize the notations introduced above) the following equality for the signs

$$
\begin{aligned}
\sigma_{1}^{m_{1}} \cdots \sigma_{n-k}^{m_{n-k}} & \operatorname{sgn}\left(g_{s}^{(n-k)}\left(z_{1}, \ldots, z_{k}\right)\right) \\
\quad & =\operatorname{sgn}\left(\bar{g}\left(z_{1}, \ldots, z_{k}, \sigma_{1} \varepsilon_{k+1} \varepsilon_{n+1}, \ldots, \sigma_{n-k} \varepsilon_{n} \varepsilon_{n+1}\right)\right)
\end{aligned}
$$

holds for any $\sigma_{1}, \ldots, \sigma_{n-k} \in\{-1,1\}$. For any $1 \leq i \leq n-k$ such that $m_{i}=0$, (1) holds also for $\sigma_{i}=0$, agreeing that $0^{0}=1$. Moreover, the following polynomial identity holds:

$$
g_{s}^{(n-k)}\left(Z_{1}, \ldots, Z_{k}\right)=s t_{k}\left(\frac{\bar{g}\left(Z_{1}, \ldots, Z_{k}, \varepsilon_{k+1} \varepsilon_{n+1}, \ldots, \varepsilon_{n} \varepsilon_{n+1}\right)}{\varepsilon_{k+1}^{m_{1}} \cdots \varepsilon_{n}^{m_{n-k}} \varepsilon_{n+1}^{s}}\right) .
$$

Now let $F$ be an algebraically closed field of zero characteristic. Take a certain $0<\eta \leq 1$ (it will be specified later). We call $k$-face $\Gamma=H_{i_{1}} \cap \cdots \cap H_{i_{n-k}}$ of the arrangement $S$ strongly singular (with respect to a polynomial $g \in F\left[X_{1}, \ldots, X_{n}\right]$ ) if $\operatorname{Var}^{\left(H_{i_{1}}, \ldots, H_{i_{n-k}}\right)}(g) \geq \eta(n-k)$. Denote by $N$ the number of strongly singular $k$-faces of $S$ with respect to $g$ (since $g$ will be fixed for the time being, in what follows we omit mentioning $g$ in this context).

\section{Multiplicative Complexity and Strongly Singular Faces}

Consider the graph (see [27] and [19]) of the gradient map $G=\left\{\left(x, \operatorname{grad}_{g}(x)\right): x \in\right.$ $\left.F^{n}\right\} \subset F^{2 n}=\left\{\left(x_{1}, \ldots, x_{n}, v_{1}, \ldots, v_{n}\right)\right\}$, so $v_{i}=\left(\partial g / \partial X_{i}\right)(x), 1 \leq i \leq n$. The notion of the degree deg was extended in [17] to constructible sets in an affine space from the usual case of closed projective sets [25], [24]. We are now able to formulate the main technical tool of this section (see Theorem 1 of [8]).

Lemma 1. For any $0 \leq k \leq n, 0<\eta \leq 1$, and an arrangement $S=H_{1} \cup \cdots \cup H_{m}$ having $N$ strongly singular $k$-faces with respect to a polynomial $g \in F\left[X_{1}, \ldots, X_{n}\right]$ over an algebraically closed zero-characteristics field $F$, the following bound holds: $\operatorname{deg} G \geq \Omega\left(N /\left(m^{(1-\eta)(n-k)} 2^{4 n}\right)\right)$. 
Proof. Without loss of generality we assume that $N \geq 1$, otherwise the lemma is trivial. We introduce a linear projection $\varphi: F^{2 n} \rightarrow F^{n}$ where $\varphi\left(X_{1}, \ldots, X_{n}, V_{1}, \ldots, V_{n}\right)=$ $\left(X_{1}, \ldots, X_{n}\right)$. We also introduce a rational map $\psi: F^{2 n} \rightarrow \mathbb{P}^{n^{2}+n-1}$ where $\mathbb{P}^{n^{2}+n-1}$ is the projective space with the coordinates $\left\{W_{i \ell}\right\}_{1 \leq i, \ell \leq n}:\left\{W_{\ell}\right\}_{1 \leq \ell \leq n}$, herewith $\psi$ is given by the formulae $W_{i \ell}=X_{i} V_{\ell}, W_{\ell}=V_{\ell}, 1 \leq i, \ell \leq n$. Thus, $\psi$ is defined for any point $(x, v) \in F^{2 n}$ such that $v \neq 0$. In fact, $\psi$ could be viewed as the composition of the following natural rational maps $F^{2 n} \rightarrow F^{n} \times\left(F^{n}-0\right) \rightarrow F^{n} \times \mathbb{P}^{n-1} \hookrightarrow \mathbb{P}^{n} \times \mathbb{P}^{n-1} \hookrightarrow$ $\mathbb{P}^{n^{2}+n-1}$, where the latter one is the Segre embedding [25], [24]. Finally, we denote by $\sigma: \mathbb{P}^{n^{2}+n-1} \rightarrow \mathbb{P}^{n-1}$ the linear projection, where $\sigma\left(\left\{W_{i \ell}\right\}:\left\{W_{\ell}\right\}\right)=\left\{W_{\ell}\right\}$. The role of $\sigma$ is to distinguish the coordinates of the gradient.

For the time being fix a strongly singular $k$-face $\Gamma=H_{i_{1}} \cap \cdots \cap H_{i_{n-k}}$ of $g$. We recall that for any point $v$ from $\Gamma$ the chosen coordinates $Y_{1}, \ldots, Y_{n-k}$ vanish at $v$, herewith $H_{i_{1}}, \ldots, H_{i_{n-k}}$ are the coordinate hyperplanes for $Y_{1}, \ldots, Y_{n-k}$. We have an expansion $\bar{g}=g_{s}+g_{s+1}+\cdots+g_{s_{1}}$, where the polynomial $g_{j} \in F\left[Z_{1}, \ldots, Z_{k}, Y_{1}, \ldots, Y_{n-k}\right]$, $s \leq j \leq s_{1}$, is homogeneous of degree $j$ with respect to the variables $Y_{1}, \ldots, Y_{n-k}$, and $g_{s} \not \equiv 0$. Let $Y_{\ell_{1}}, \ldots, Y_{\ell_{p}}, p \geq \eta(n-k)$, occur in $\operatorname{lm}\left(g_{s}\right)=\alpha Z_{1}^{m_{1}^{\prime}} \cdots Z_{k}^{m_{k}^{\prime}} Y_{1}^{m_{1}} \cdots Y_{n-k}^{m_{n-k}}$. Remember that $m_{1}, \ldots, m_{n-k}$ do not depend on the coordinates $Z_{1}, \ldots, Z_{k}$, thereby on a particular point $v$ from $\Gamma$; also $g_{s}^{(n-k)} \in F\left[Z_{1}, \ldots, Z_{k}\right]$ is the coefficient at $Y_{1}^{m_{1}} \cdots Y_{n-k}^{m_{n-k}}$ of the expansion of $g_{s}$, herewith $\operatorname{lm}\left(g_{s}^{(n-k)}\right)=\alpha Z_{1}^{m_{1}^{\prime}} \cdots Z_{k}^{m_{k}^{\prime}}$.

For the sake of simplifying the notations, we make a linear transformation of the coordinates $X_{1}, \ldots, X_{n}$ into $Z_{1}, \ldots, Z_{k}, Y_{1}, \ldots, Y_{n-k}$ and we also apply the same linear transformation to the coordinates $V_{1}, \ldots, V_{n}$ (keeping the same notation for them). Then in the new coordinates

$$
\begin{array}{r}
G=\left\{\left(z_{1}, \ldots, z_{k}, y_{1}, \ldots, y_{n-k}, v_{1}, \ldots, v_{n}\right): v_{i}=\frac{\partial \bar{g}}{\partial Z_{i}}\left(z_{1}, \ldots, z_{k}, y_{1}, \ldots, y_{n-k}\right),\right. \\
\left.1 \leq i \leq k ; \quad v_{j+k}=\frac{\partial \bar{g}}{\partial Y_{j}}\left(z_{1}, \ldots, z_{k}, y_{1}, \ldots, y_{n-k}\right), \quad 1 \leq j \leq n-k\right\}
\end{array}
$$

and $\psi$ is given by the same formulae $W_{i \ell}=Z_{i} V_{\ell}, W_{\ell}=V_{\ell}$ as above.

For a fixed point $v \in \Gamma$ consider the $(n-1)$-dimensional plane $\mathcal{P}=\mathcal{P}_{v}=\left\{W_{i \ell}=\right.$ $\left.W_{j \ell}=0 ; 1 \leq i \leq k, 1 \leq j \leq n-k, 1 \leq \ell \leq n\right\}=\psi\left(\varphi^{-1}(v)\right) \subset \mathbb{P}^{n^{2}+n-1}$. The following lemma is similar to Lemma 1 of [8].

Lemma 2. It holds that $\operatorname{dim}(\sigma(\overline{\psi(G)} \cap \mathcal{P})) \geq \eta(n-k)-1$. Moreover, the linear (coordinate) functions $W_{\ell_{1}}, \ldots, W_{\ell_{p}}$ are algebraically independent on $\overline{\psi(G)} \cap \mathcal{P}$.

Proof. Suppose the contrary. Then there exists a homogeneous polynomial $0 \not \equiv h \in$ $F\left[W_{\ell_{1}}, \ldots, W_{\ell_{p}}\right]$ which vanishes $h\left(w_{\ell_{1}}, \ldots, w_{\ell_{p}}\right)=0$ for any point $\left(\{0\}:\left\{w_{\ell}\right\}\right) \in$ $\overline{\psi(G)} \cap \mathcal{P}$.

Let an integer $\theta_{1}$ be greater than any exponent in the terms occurring in the polynomial $g_{s}^{(n-k)}$. Then the value of the linear function $u_{1}+\theta_{1} u_{2}+\theta_{1}^{2} u_{3}+\cdots+\theta_{1}^{k-1} u_{k}$ at the exponent vector of the minimal term $\alpha Z_{1}^{m_{1}^{\prime}} \cdots Z_{k}^{m_{k}^{\prime}}$ of the polynomial $g_{s}^{(n-k)}$ (we denote this value by $\theta=m_{1}^{\prime}+\theta_{1} m_{2}^{\prime}+\cdots+\theta_{1}^{k-1} m_{k}^{\prime}$ ) is less than the value of this linear function for any other term of $g_{s}^{(n-k)}$. 
Take a point $\left(z_{1}, \ldots, z_{k}, y_{1}, \ldots, y_{n-k}\right) \in F^{n}$. Consider an algebraic curve $\left\{t_{\lambda}=\right.$ $\left.\left(\lambda z_{1}, \lambda^{\theta_{1}} z_{2}, \ldots, \lambda^{\theta_{1}^{k-1}} z_{k}, \lambda^{\theta+1} y_{1}, \ldots, \lambda^{\theta+1} y_{n-k}\right)\right\}_{\lambda \in F} \subset F^{n}$ passing through the points $(0, \ldots, 0)$ and $\left(z_{1}, \ldots, z_{k}, y_{1}, \ldots, y_{n-k}\right)$. Then

$$
\begin{aligned}
\frac{\partial \bar{g}}{\partial Y_{j}}\left(t_{\lambda}\right) & =\left(\frac{\partial g_{s}}{\partial Y_{j}}+\frac{\partial g_{s+1}}{\partial Y_{j}}+\cdots+\frac{\partial g_{s_{1}}}{\partial Y_{j}}\right)\left(t_{\lambda}\right) \\
& =\lambda^{(s-1)(\theta+1)+\theta}\left(m_{j} \alpha z_{1}^{m_{1}^{\prime}} \cdots z_{k}^{m_{k}^{\prime}} y_{1}^{m_{1}} \cdots y_{j}^{m_{j}-1} \cdots y_{n-k}^{m_{n-k}}+\lambda \widetilde{g}^{(j)}\right),
\end{aligned}
$$

where $\tilde{g}^{(j)} \in F\left[\lambda, z_{1}, \ldots, z_{k}, y_{1}, \ldots, y_{n-k}\right], 1 \leq j \leq n-k$. Similarly, $\left(\partial \bar{g} / \partial Z_{i}\right)\left(t_{\lambda}\right)=$ $\lambda^{s(\theta+1)} \widehat{g}^{(i)}$, where $\hat{g}^{(i)} \in F\left[\lambda, z_{1}, \ldots, z_{k}, y_{1}, \ldots, y_{n-k}\right]$.

Hence the point

$$
\begin{gathered}
\left(\left\{\lambda^{\theta_{1}^{i-1}} z_{i} \cdot \frac{\partial \bar{g}}{\partial Z_{\ell}}\left(t_{\lambda}\right)\right\}:\left\{\lambda^{\theta+1} y_{j} \cdot \frac{\partial \bar{g}}{\partial Z_{\ell}}\left(t_{\lambda}\right)\right\}:\left\{\lambda^{\theta_{1}^{i-1}} \cdot z_{i} \cdot \frac{\partial \bar{g}}{\partial Y_{q}}\left(t_{\lambda}\right)\right\}:\right. \\
\left.\left\{\lambda^{\theta+1} y_{j} \cdot \frac{\partial \bar{g}}{\partial Y_{q}}\left(t_{\lambda}\right)\right\}: \frac{\partial \bar{g}}{\partial Z_{1}}\left(t_{\lambda}\right): \cdots: \frac{\partial \bar{g}}{\partial Z_{k}}\left(t_{\lambda}\right): \frac{\partial \bar{g}}{\partial Y_{1}}\left(t_{\lambda}\right): \cdot: \frac{\partial \bar{g}}{\partial Y_{n-k}}\left(t_{\lambda}\right)\right) \\
1 \leq i, \ell \leq k ; 1 \leq j, q \leq n-k \\
=\psi\left(t_{\lambda}, \frac{\partial \bar{g}}{\partial Z_{1}}\left(t_{\lambda}\right), \ldots, \frac{\partial \bar{g}}{\partial Z_{k}}\left(t_{\lambda}\right), \frac{\partial \bar{g}}{\partial Y_{1}}\left(t_{\lambda}\right), \ldots, \frac{\partial \bar{g}}{\partial Y_{n-k}}\left(t_{\lambda}\right)\right) \in \psi(G)
\end{gathered}
$$

(provided that this point of the projective space is defined, i.e., not all $\left(\partial \bar{g} / \partial Z_{1}\right)\left(t_{\lambda}\right), \ldots$, $\left(\partial \bar{g} / \partial Z_{k}\right)\left(t_{\lambda}\right),\left(\partial \bar{g} / \partial Y_{1}\right)\left(t_{\lambda}\right), \ldots,\left(\partial \bar{g} / \partial Y_{n-k}\right)\left(t_{\lambda}\right)$ vanish). Divide all the coordinates of this point over their common factor $\lambda^{(s-1)(\theta+1)+\theta}$ and after that plug $\lambda=0$. Then the resulting point $\left(\{0\}: 0: \cdots: 0: m_{1} z_{1}^{m_{1}^{\prime}} \cdots z_{k}^{m_{k}^{\prime}} y_{1}^{m_{1}-1} y_{2}^{m_{2}} \cdots y_{n-k}^{m_{n-k}}: \cdots: m_{n-k} z_{1}^{m_{1}^{\prime}} \cdots z_{k}^{m_{k}^{\prime}}\right.$ $\left.y_{1}^{m_{1}} \cdots y_{n-k}^{m_{n-k}-1}\right) \in \overline{\psi(G)}$.

Because $m_{\ell_{1}}, \ldots, m_{\ell_{p}}$ are positive, the above supposition implies that $h\left(y^{M} / y_{\ell_{1}}, \ldots\right.$, $\left.y^{M} / y_{\ell_{p}}\right)=0$ for arbitrary $y_{\ell_{1}}, \ldots, y_{\ell_{p}} \in F$ where $y^{M}=y_{\ell_{1}}^{m_{\ell_{1}}} \cdots y_{\ell_{p}}^{m_{\ell_{p}}}$. This means that $h\left(Y^{M} / Y_{\ell_{1}}, \ldots, Y^{M} / Y_{\ell_{p}}\right) \equiv 0$ for the variables $Y_{\ell_{1}}, \ldots, Y_{\ell_{p}}$. We prove that the latter is impossible. When $p=1$ this is obvious. Assume that $p \geq 2$. Then for any two distinct integer multi-indices $\left(i_{1}, \ldots, i_{p}\right) \neq\left(j_{1}, \ldots, j_{p}\right)$ we have $\left(Y^{M} / Y_{\ell_{1}}\right)^{i_{1}} \ldots\left(Y^{M} / Y_{\ell_{p}}\right)^{i_{p}} \neq$ $\left(Y^{M} / Y_{\ell_{1}}\right)^{j_{1}} \cdots\left(Y^{M} / Y_{\ell_{p}}\right)^{j_{p}}$. Indeed, otherwise $\left(Y^{M} / Y_{\ell_{1}}\right)^{i_{1}-j_{1}} \cdots\left(Y^{M} / Y_{\ell_{p}}\right)^{i_{p}-j_{p}}=1$, i.e., $Y^{M\left(i_{1}-j_{1}+\cdots+i_{p}-j_{p}\right)}=Y_{\ell_{1}}^{i_{1}-j_{1}} \cdots Y_{\ell_{p}}^{i_{p}-j_{p}}$, therefore the multi-indices $\left(i_{1}-j_{1}, \ldots, i_{p}-\right.$ $\left.j_{p}\right)=\left(i_{1}-j_{1}+\cdots+i_{p}-j_{P}\right) M$ coincide, but the sums of the coordinates in both multi-indices differ by the factor of $m_{\ell_{1}}+\cdots+m_{\ell_{p}} \geq 2$.

The contradiction obtained completes the proof of Lemma 2.

Now we return to the proof of Lemma 1. Observe that $\overline{\psi(G)} \cap \mathcal{P} \subset \mathbb{P}^{n^{2}+n-1}$ (where $\mathcal{P}=\mathcal{P}_{\nu}$ for an arbitrary point $\nu \in \Gamma$, see above) is a closed projective variety and the projection $\sigma$ is defined everywhere on this variety, so being a regular map, hence $\sigma(\overline{\psi(G)} \cap \mathcal{P}) \subset \mathbb{P}^{n-1}$ is a closed projective variety (see [25] and [24]).

There exists a subspace $B \subset \mathbb{P}^{n-1}$ with the dimension $\operatorname{dim} B=\lfloor n-\eta(n-k)\rfloor$ such that $\operatorname{dim}\left(\overline{\psi(G)} \cap \sigma^{-1}(B)\right) \leq n-\eta(n-k)+1$ (actually, almost any subspace satisfies this property). This follows from the theorem of the dimension of fibers [24], applying it to the rational dominating map $\sigma: \overline{\psi(G)} \rightarrow \overline{\sigma(\overline{\psi(G)})}$ and taking into account that $\operatorname{dim} \overline{\psi(G)}=\operatorname{dim} G=n$. 
Since the intersection of two closed projective varieties of the complement dimensions (see Lemma 2) $B \cap \sigma(\overline{\psi(G)} \cap \mathcal{P})$ is not empty [25], [24], we conclude that $\sigma^{-1}(B) \cap$ $\overline{\psi(G)} \cap \mathcal{P} \neq \emptyset$, for any point $v$ from any strongly singular $k$-face $\Gamma$. Varying $\mathcal{P}=\mathcal{P}_{\nu}$ for different points $v$ from $\Gamma$, the latter implies, in particular, that $\operatorname{dim}\left(\sigma^{-1}(B) \cap \overline{\psi(G)}\right) \geq k$.

Therefore, the constructible set $U=\varphi\left(\psi^{-1}\left(\sigma^{-1}(B) \cap \overline{\psi(G)}\right)\right) \subset F^{n}$ contains all strongly singular $k$-faces $\Gamma$. Observe that for any point $u \in \mathbb{P}^{n^{2}+n-1}$ the set $\varphi\left(\psi^{-1}(u)\right)$ consists of a single point when $u \in \psi\left(F^{2 n}\right)$ or else is empty. Thus, $\varphi \psi^{-1}: \overline{\psi\left(F^{2 n}\right)} \rightarrow F^{n}$ is a rational surjective map [25]. Hence $\operatorname{dim} U \leq \operatorname{dim}\left(\sigma^{-1}(B) \cap \overline{\psi(G)}\right) \leq n-\eta(n-k)+1$.

For each strongly singular $k$-face $\Gamma=H_{i_{1}} \cap \cdots \cap H_{i_{n-k}}$ successively choose $j_{1}, j_{2}, \ldots \in$ $\left\{i_{1}, \ldots, i_{n-k}\right\}$, such that for every $\ell \geq 0$ we have $\operatorname{dim}\left(U \cap H_{j_{1}} \cap \cdots \cap H_{j_{\ell}} \cap H_{j_{\ell+1}}\right) \leq$ $\operatorname{dim}\left(U \cap H_{j_{1}} \cap \cdots \cap H_{j_{\ell}}\right)-1$ while $\operatorname{dim}\left(U \cap H_{j_{1}} \cdots \cap H_{j_{\ell}}\right)>k$. After at most $q \leq n-\eta(n-k)+1-k$ steps we reach $j_{1}, \ldots, j_{q}$ for which $\operatorname{dim}\left(U \cap H_{j_{1}} \cap \cdots \cap H_{j_{q}}\right)=k$, thus $\Gamma$ is an irreducible component of $U \cap H_{j_{1}} \cap \cdots \cap H_{j_{q}}$. Take also an $(n-k)$-dimensional plane $Q \subset F^{n}$ transversal to all $k_{1}$-faces of $S$ for all $0 \leq k_{1} \leq n$ and to all irreducible components of $U \cap H_{j_{1}} \cap \cdots \cap H_{j_{q}}$ for all $j_{1}, \ldots, j_{q}$. Then the point $Q \cap \Gamma$, being an irreducible component of zero-dimensional variety $U \cap H_{j_{1}} \cap \cdots \cap H_{j_{q}} \cap Q$, does not belong to any other $k$-faces except $\Gamma$.

Consider the constructible sets $\mathcal{H}_{i}=\psi\left(\varphi^{-1}\left(H_{i}\right)\right)$ and $\mathcal{Q}=\psi\left(\varphi^{-1}(Q)\right) \subset \mathbb{P}^{n^{2}+n-1}$, $1 \leq i \leq m$. Consider also

$$
\mathcal{U}=\mathcal{U}_{j_{1}, \ldots j_{q}}=\sigma^{-1}(B) \cap \overline{\psi(G)} \cap \mathcal{H}_{j_{1}} \cap \cdots \cap \mathcal{H}_{j_{q}} \cap \mathcal{Q} .
$$

Then $\varphi\left(\psi^{-1}(\mathcal{U})\right)=U \cap H_{j_{1}} \cap \cdots \cap H_{j_{q}} \cap Q=\left\{u_{1}, \ldots, u_{\kappa}\right\} \subset F^{n}$ is a finite collection of points. Therefore, every irreducible component of $\mathcal{U}$ is contained in one of the pairwise disjoint $(n-1)$-dimensional planes $\psi\left(\varphi^{-1}\left(u_{1}\right)\right), \ldots, \psi\left(\varphi^{-1}\left(u_{\kappa}\right)\right) \subset \mathbb{P}^{n^{2}+n-1}$, since the image of this irreducible component under the rational map $\varphi \psi^{-1}: \overline{\psi\left(F^{2 n}\right)} \rightarrow F^{n}$, being a subset of $\left\{u_{1}, \ldots, u_{\kappa}\right\}$, should be a point; moreover, each of these planes contains a certain component of $\mathcal{U}$.

Thus, $\operatorname{deg}(\mathcal{U})=\operatorname{deg}\left(\mathcal{U}_{j_{1}, \ldots, j_{q}}\right) \geq \kappa$; we define the degree of a constructible set as the degree of its projective closure $\overline{\mathcal{U}}$ [24], [25], i.e., the sum of the degrees of irreducible components of $\overline{\mathcal{U}}$.

Taking the sum of the latter inequalities over all $1 \leq j_{1}, \ldots, j_{q} \leq m, q \leq(n-k)(1-$ $\eta)+1$, and observing that each strongly singular $k$-face $\Gamma$ gives a contribution into the right-hand side of one of these inequalities, we conclude that

$$
\sum_{1 \leq j_{1}, \ldots, j_{q} \leq m ; q \leq(n-k)(1-\eta)+1} \operatorname{deg}\left(\mathcal{U}_{j_{1}, \ldots, j_{q}}\right) \geq N .
$$

Another method for bounding from below the degree of a variety passing through a given set of points can be found in [28], but this method is not applicable here. To bound $\operatorname{deg}\left(\mathcal{U}_{j_{1}, \ldots, j_{q}}\right)$ from above, we rely on the following lemma.

Lemma 3. Let an affine Zariski closed set $\mathcal{V} \subset F^{2 n}$. Then $\operatorname{deg}(\psi(\mathcal{V})) \leq 2^{2 n} \operatorname{deg}(\mathcal{V})$.

Proof (see the proof of Theorem 1 of [8]). Since $\operatorname{dim}(\overline{\psi(\mathcal{V})}-\psi(\mathcal{V}))<\operatorname{dim}(\psi(\mathcal{V}))$, there is a subspace $R \subset \mathbb{P}^{n^{2}+n-1}$ with $\operatorname{dim} R=n^{2}+n-1-\operatorname{dim} \psi(\mathcal{V})$ for which $R \cap \psi(\mathcal{V})$ consists of $\operatorname{deg} \psi(\mathcal{V})=\operatorname{deg} \overline{\psi(\mathcal{V})}$ points (in fact, almost any subspace has 
$\operatorname{deg} \psi(\mathcal{V})$ common points with $\overline{\psi(\mathcal{V})}$, and almost any subspace has an empty intersection with $\overline{\psi(\mathcal{V})}-\psi(\mathcal{V}))$. Because $\psi\left(\psi^{-1}(R) \cap \mathcal{V}\right)=R \cap \psi(\mathcal{V})$, the degree deg $\psi(\mathcal{V})$ does not exceed the number of irreducible components of the variety $\psi^{-1}(R) \cap \mathcal{V}$, which in its turn is less than or equal to $\operatorname{deg}\left(\psi^{-1}(R) \cap \mathcal{V}\right)$. Then we apply the Bezout inequality $\operatorname{deg}\left(\psi^{-1}(R) \cap \mathcal{V}\right) \leq \operatorname{deg}\left(\psi^{-1}(R)\right) \cdot \operatorname{deg} \mathcal{V}$ which was proved for affine closed sets in [17], rather than for the usual case of projective closed varieties with complete intersection [24], [25]. The closedness of $\psi^{-1}(R)$ follows from the next paragraph.

It remains to bound $\operatorname{deg}\left(\psi^{-1}(R)\right)$. If $R$ is determined by several linear equations of the form $\sum_{1 \leq i, \ell \leq n} \alpha_{i \ell} W_{i \ell}+\sum_{1<\ell \leq n} \beta_{\ell} W_{\ell}=0$, then $\psi^{-1}(R)$ is determined by the quadratic equations $\sum_{1 \leq i, \ell \leq n} \alpha_{i \ell} X_{i} V_{\ell}+\sum_{1 \leq \ell \leq n} \beta_{\ell} V_{\ell}=0$. One can choose $2 n$ suitable linear combination of these equations $\zeta_{1}, \ldots, \zeta_{2 n} \in F\left[X_{1}, \ldots, X_{n}, V_{1}, \ldots, V_{n}\right]$ such that the irreducible components of the variety $\left\{\zeta_{1}=\cdots=\zeta_{2 n}=0\right\} \subset F^{2 n}$ contain all the irreducible components of $\psi^{-1}(R)$ and in addition, perhaps, a few points, being its zero-dimensional components (see also [6]). Hence $\operatorname{deg}\left(\psi^{-1}(R)\right) \leq 2^{2 n}$ again due to the Bezout inequality. This completes the proof of Lemma 3.

Returning to bounding $\operatorname{deg}\left(\mathcal{U}_{j_{1}, \ldots, j_{q}}\right)$ from above, we note that $\mathcal{H}_{j_{1}} \cap \cdots \cap \mathcal{H}_{j_{q}} \cap \mathcal{Q}=$ $\psi\left(\varphi^{-1}\left(H_{j_{1}} \cap \cdots \cap H_{j_{q}} \cap Q\right)\right)($ see $(2))$ and $\mathcal{H}=\varphi^{-1}\left(H_{j_{1}} \cap \cdots \cap H_{j_{q}} \cap Q\right) \subset F^{2 n}$ is a plane, so of degree 1. Applying Lemma 3 we obtain the bound

$$
\operatorname{deg}(\psi(\mathcal{H})) \leq 2^{2 n}
$$

For every $1 \leq i \leq n$ consider a principle affine Zariski open chart $\mathcal{A}_{\ell}=\left\{W_{\ell} \neq 0\right\} \subset$ $\mathbb{P}^{n^{2}+n-1}$ and denote $\mathcal{A}=\bigcup_{1 \leq \ell \leq n} \mathcal{A}_{\ell}$. Observe that $\sigma^{-1}(B) \subset \mathcal{A}$ is closed in $\mathcal{A}$.

We also show that $\mathcal{H}_{j_{1}} \cap \cdots \cap \mathcal{H}_{j_{q}} \cap \mathcal{Q}=\psi(\mathcal{H}) \subset \psi\left(F^{2 n}\right) \subset \mathcal{A}$ is closed in $\mathcal{A}$. Indeed, $\mathcal{H}$ is given by a system of linear equations $\left\{h_{t}=\sum_{1 \leq i \leq n} \gamma_{t i} X_{i}+\gamma_{t 0}=0\right\}_{t}$ which depend only on $X_{1}, \ldots, X_{n}$. We claim that

$$
\begin{aligned}
\psi(\mathcal{H})=\mathcal{A} & \cap\left\{\sum_{1 \leq i \leq n} \gamma_{t i} W_{i \ell}+\gamma_{t 0} W_{\ell}=0\right\}_{t, 1 \leq \ell \leq n} \\
& \cap\left\{W_{i \ell_{1}} W_{\ell_{2}}=W_{i \ell_{2}} W_{\ell_{1}}\right\}_{1 \leq i, \ell_{1}, \ell_{2} \leq n} .
\end{aligned}
$$

The inclusion $\subset$ is obvious. To prove the inverse inclusion take a point $\left\{w_{i \ell}\right\}_{i, \ell}:\left\{w_{\ell}\right\}_{\ell}$ from the set at the right-hand side. Then $w_{\ell_{0}} \neq 0$ for a certain $1 \leq \ell_{0} \leq n$. From the equalities $w_{i \ell_{1}} w_{\ell_{2}}=w_{i \ell_{2}} w_{\ell_{1}}$ we get that $\left\{w_{i \ell}\right\}_{i, \ell}:\left\{w_{\ell}\right\}_{\ell}=\psi\left(w_{1 \ell_{0}} / w_{\ell_{0}}, \cdots, w_{n \ell_{0}} / w_{\ell_{0}}\right.$, $\left.w_{1}, \ldots, w_{n}\right)$. Finally, the equalities $\left\{\sum_{1 \leq i \leq n} \gamma_{t i} w_{i \ell_{0}}+\gamma_{t 0} w_{\ell_{0}}=0\right\}_{t}$ entail that $\left(w_{1 \ell_{0}} / w_{\ell_{0}}\right.$, $\left.\ldots, w_{n \ell_{0}} / w_{\ell_{0}}, w_{1}, \ldots, w_{n}\right) \in \mathcal{H}$, which proves the inverse inclusion of the claim and thereby the closedness of $\psi(\mathcal{H})$ in $\mathcal{A}$.

Let $\mathcal{U}=\sigma^{-1}(B) \cap \overline{\psi(G)} \cap \psi(\mathcal{H})=\bigcup_{j} \mathcal{U}_{j} \subset \mathcal{A}$ (see (2)) be the decomposition of $\mathcal{U}$, being the intersection of three Zariski closed in $\mathcal{A}$ subsets, as was just proved, into its irreducible components $\mathcal{U}_{j}$. For every $1 \leq i \leq n$ we have the induced decomposition of the intersection

$$
\left(\sigma^{-1}(B) \cap \mathcal{A}_{i}\right) \cap\left(\overline{\psi(G)} \cap \mathcal{A}_{i}\right) \cap\left(\psi(\mathcal{H}) \cap \mathcal{A}_{i}\right)=\bigcup_{j}\left(\mathcal{U}_{j} \cap \mathcal{A}_{i}\right)
$$


of three Zariski closed affine sets (in $\mathcal{A}_{i}$ ) into its irreducible components $\mathcal{U}_{j} \cap \mathcal{A}_{i}$, provided that $\mathcal{U}_{j} \cap \mathcal{A}_{i} \neq \varnothing$. Moreover, in the latter case the closure $\overline{\mathcal{U}_{j} \cap \mathcal{A}_{i}}=\overline{\mathcal{U}_{j}} \subset \mathbb{P}^{n^{2}+n-1}$ because $\mathcal{A}_{i}$ is open in $\mathbb{P}^{n^{2}+n-1}$, in particular, $\operatorname{deg}\left(\mathcal{U}_{j} \cap \mathcal{A}_{i}\right)=\operatorname{deg} \mathcal{U}_{j}$. Applying the affine version of the Bezout inequality [17], we obtain

$$
\begin{aligned}
\sum_{j} \operatorname{deg} \mathcal{U}_{j} & \leq \operatorname{deg}\left(\sigma^{-1}(B) \cap \mathcal{A}_{i}\right) \operatorname{deg}\left(\overline{\psi(G)} \cap \mathcal{A}_{i}\right), \\
\operatorname{deg}\left(\psi(\mathcal{H}) \cap \mathcal{A}_{i}\right) & \leq \operatorname{deg} \overline{\psi(G)} \cdot \operatorname{deg} \psi(\mathcal{H}),
\end{aligned}
$$

where the summation ranges over $j$ for which $\mathcal{U}_{j} \cap \mathcal{A}_{i} \neq \emptyset$. Summing up these inequalities for all $1 \leq i \leq n$, we conclude

$$
\operatorname{deg} \mathcal{U}=\sum_{j} \operatorname{deg} \mathcal{U}_{j} \leq n \cdot \operatorname{deg} \overline{\psi(G)} \cdot \operatorname{deg} \psi(\mathcal{H}),
$$

which together with the bounds (3) and (4) gives the inequality

$$
N \leq\left(\begin{array}{c}
m \\
\lfloor(n-k)(1-\eta)\rfloor+1
\end{array}\right) \cdot(n-k)(1-\eta) n \cdot \operatorname{deg} \overline{\psi(G)} \cdot 2^{2 n},
$$

hence taking into account the inequality $\operatorname{deg} \overline{\psi(G)} \leq 2^{2 n} \operatorname{deg} G$ following from Lemma 3, we finally get

$$
\operatorname{deg} G \geq \Omega\left(\frac{N}{m^{(n-k)(1-\eta)} 2^{4 n}}\right),
$$

that completes the proof of Lemma 1.

Corollary (see Corollary 1 of [8]). Let a polynomial $g \in F\left[X_{1}, \ldots, X_{n}\right]$ have $N$ strongly singular $k$-faces in an arrangement $H_{1} \cup \cdots \cup H_{m} \subset F^{n}$. Then the multiplicative complexity $C(g) \geq 1 / 3(\log N-(n-k)(1-\eta) \log m-4 n-$ const $)$.

The results from [27] and [1] imply that $\operatorname{deg} G \leq 2^{3 C(g)}$, then make use of Lemma 1 .

\section{Lower Bound for Randomized Computation Trees}

Recall (see, e.g., [2]) that in the computation tree (CT) testing polynomials are computed along paths using elementary arithmetic operations. In particular, for a testing polynomial $f_{i} \in \mathbb{R}\left[X_{1}, \ldots, X_{n}\right]$ at the level $i$ (assuming that the root has the zero level) we have the obvious bound on its complexity, a fortiori multiplicative complexity $C\left(f_{i}\right) \leq i$. Under RCT (see [20] and [21]) we mean a collection of CT $T=\left\{T_{\alpha}\right\}$ and a probabilistic vector $p_{\alpha} \geq 0, \sum_{\alpha} p_{\alpha}=1$, such that CT $T_{\alpha}$ is chosen with probability $p_{\alpha}$. The depth of an RCT (treated as its complexity) is defined as the maximum of the depths of all $T_{\alpha}$ 's (actually the equivalent complexity classes that are obtained to define the depth of an RCT as the expectation of the depths of $T_{\alpha}$ 's [20]). The main requirement is that for any input an RCT gives a correct output with probability $1-\gamma>\frac{1}{2}(\gamma$ is called the error probability of the RCT). 
For a hyperplane $H \subset \mathbb{R}^{n}$ by $H^{+} \subset \mathbb{R}^{n}$ denote the closed halfspace $\left\{L_{H} \geq 0\right\}$, where $L_{H}$ is a certain linear function with the zero set $H$. For a family of hyperplanes $H_{1}, \ldots, H_{m}$ the intersection $S^{+}=\bigcap_{1 \leq i \leq m} H_{i}^{+}$is called a polyhedron. An intersection $\Gamma=H_{i_{1}} \cap \cdots \cap H_{i_{n-k}}$ is called the $k$-face of $S^{+}$if for each $1 \leq l \leq n-k+1$ we have $\operatorname{dim}\left(H_{i_{l}} \cap \cdots \cap H_{i_{n-k}}\right)=\operatorname{dim}\left(H_{i_{l}} \cap \cdots \cap H_{i_{n-k}} \cap S^{+}\right)=k+l-1$ (then clearly $H_{i_{l}} \cap \cdots \cap H_{i_{n-k}}$ is the $(k+l-1)$-face of $S^{+}$). Recall (see Section 1) that $\Gamma$ is the $k$-face of the arrangement $S=\bigcup_{1 \leq i \leq m} H_{i}$ if $\operatorname{dim} \Gamma=k$.

Now we are able to formulate the main result of this paper.

Theorem. For any positive constants $c, c_{1}, c_{2}$ there exists $c_{0}>0$ satisfying the following. Let for some $k \leq\left(1-c_{1}\right) n$ an arrangement $\mathcal{S}=S=\bigcup_{1 \leq i \leq m} H_{i}$ or a polyhedron $\mathcal{S}=S^{+}=\bigcap_{1 \leq i \leq m} H_{i}^{+}$have at least $c_{2}\left(m^{c(n-k)}\right) k$-faces. Then for any $R C T$ recognizing $\mathcal{S}$, its depth is greater than $c_{0}(n \log m)$.

For a family of polynomials $f_{1}, \ldots, f_{t} \in \mathbb{R}\left[X_{1}, \ldots, X_{n}\right]$ we define $\operatorname{Var}^{(\Gamma)}\left(f_{1}, \ldots, f_{t}\right)$ to be the number of variables among $Y_{1}, \ldots, Y_{n-k}$ (we utilize the notations introduced in Section 1) which occur in at least one of the leading terms $\operatorname{lm}\left(f_{1, s_{1}}\right), \ldots, \operatorname{lm}\left(f_{t, s_{t}}\right)$, where $H_{i_{1}}, \ldots, H_{i_{n-k}}$ are the coordinate hyperplanes of the coordinates $Y_{1}, \ldots, Y_{n-k}$, respectively; $\bar{f}_{j}\left(Z_{1}, \ldots, Z_{k}, Y_{1}, \ldots, Y_{n-k}\right)=f_{j}\left(X_{1}, \ldots, X_{n}\right)$ and $\bar{f}_{j}=f_{j, s_{j}}+f_{j, s_{j}+1}+$ $\ldots$, herewith $f_{j, l}$ is homogeneous with respect to the variables $Y_{1}, \ldots, Y_{n-k}$ of degree $l$ and $f_{j, s_{j}} \not \equiv 0,1 \leq j \leq t$. Because the expansion into the homogeneous components $\bar{f}_{1} \cdots \bar{f}_{t}=\left(f_{1, s_{1}} \cdots f_{t, s_{t}}\right)+\cdots$ starts with $f_{1, s_{1}} \cdots f_{t, s_{t}}$, we have $\operatorname{lm}\left(f_{1, s_{1}} \cdots f_{t, s_{t}}\right)=$ $\operatorname{lm}\left(f_{1, s_{1}}\right) \cdots \operatorname{lm}\left(f_{t, s_{t}}\right)$ and hence $\operatorname{Var}^{\left(H_{i_{1}}, \ldots, H_{i_{n-k}}\right)}\left(f_{1} \cdots f_{t}\right)=\operatorname{Var}^{(\Gamma)}\left(f_{1} \cdots f_{t}\right)=\operatorname{Var}^{(\Gamma)}$ $\left(f_{1}, \ldots, f_{t}\right)$.

For any $C T T_{1}$ we denote by $\operatorname{Var}^{(\Gamma)}\left(T_{1}\right)=\operatorname{Var}^{\left(H_{i_{1}}, \ldots, H_{i_{n-k}}\right)}\left(T_{1}\right)$ the maximum of the $\operatorname{Var}^{(\Gamma)}\left(f_{1} \cdots f_{t}\right)$ taken over all the paths of $T_{1}$, whose $f_{1}, \ldots, f_{t}$ are testing polynomials along the path.

The following lemma is similar to Lemma 1 of [12] and [11], but differs from it due to the different definition of the leading term $l m$.

\section{Lemma 4. Let $T=\left\{T_{\alpha}\right\}$ be an RCT recognizing}

(a) an arrangement $S=\bigcup_{1 \leq i \leq m} H_{i}$ such that $\Gamma=H_{i_{1}} \cap \cdots \cap H_{i_{n-k}}$ is a $k$-face of $S$, or

(b) a polyhedron $S^{+}=\bigcap_{1 \leq i \leq m} H_{i}^{+}$such that $\Gamma=\bigcap_{1 \leq j \leq n-k} H_{i_{j}}$ is a $k$-face of $S^{+}$ (so, see above, for each $1 \leq l \leq n-k+1$ we have $\operatorname{dim}\left(\bigcap_{l \leq j \leq n-k} H_{i_{j}}\right)=$ $\left.\operatorname{dim}\left(\bigcap_{l \leq j \leq n-k} H_{i_{j}} \cap S^{+}\right)=k+l-1\right)$

with error probability $\gamma<\frac{1}{2}$. Then $\operatorname{Var}^{\left(H_{i_{1}}, \ldots, H_{i_{n-k}}\right)}\left(T_{\alpha}\right) \geq(1-2 \gamma)^{2}(n-k)$ for a fraction of $(1-2 \gamma) /(2-2 \gamma)$ of all $T_{\alpha}$ 's.

Proof of Lemma 4. Choose the coordinates $Z_{1}, \ldots, Z_{k}, Y_{1}, \ldots, Y_{n-k}$ such that $Z_{1}$, $\ldots, Z_{k}$ are the coordinates in $\Gamma$ and $H_{i_{1}}, \ldots, H_{i_{n-k}}$ are the coordinate hyperplanes of $Y_{1}, \ldots, Y_{n-k}$, respectively (see Section 1 ), which satisfy the following properties. The origin $\underbrace{(0, \ldots, 0)}_{n}$ of this coordinates system $Z_{1}, \ldots, Z_{k}, Y_{1}, \ldots, Y_{n-k}$ does not lie in 
any $l$-face with $l<k$ and, besides, in case (b) $(0, \ldots, 0)$ belongs to the polyhedron $S^{+}$. Also we require that for any testing polynomial $f$ from any CT $T_{\alpha}$ the inequality $f_{s}^{(n-k)} \underbrace{(0, \ldots, 0)}_{k} \neq 0$ holds (recall that $f_{s}^{(n-k)} \neq 0$ depends only on $H_{i_{1}}, \ldots, H_{i_{n-k}}$ and $f=f_{s}+f_{s+1}+\cdots$ where $f_{j}$ is homogeneous with respect to the variables $Y_{1}, \ldots, Y_{n-k}$ of degree $j$, see Section 1).

Observe that RCT $T$ treated over the field $\mathbb{R}_{n+1}$ recognizes the completion $S^{\left(\mathbb{R}_{n+1}\right)} \subset$ $\left(\mathbb{R}_{n+1}\right)^{n}$ (resp. $S^{+\left(\mathbb{R}_{n+1}\right)}$ ) due to the Tarski transfer principle (see Section 1). For the sake of simplicity of notations we keep the notation $S$ (resp. $S^{+}$) for the completions.

(a) Consider the point $E=(\underbrace{0, \ldots, 0}_{k}, \varepsilon_{k+1} \varepsilon_{n+1}, \ldots, \varepsilon_{n} \varepsilon_{n+1})$ and the points $E_{i}^{(0)}=$
$(\underbrace{0, \ldots, 0}_{k}, \varepsilon_{k+1} \varepsilon_{n+1}, \ldots, \varepsilon_{k+i-1} \varepsilon_{n+1}, 0, \varepsilon_{k+i+1} \varepsilon_{n+1}, \ldots, \varepsilon_{n} \varepsilon_{n+1}), 1 \leq i \leq n-k$.

Then the point $E \notin S$ (because of the choice of the origin of the coordinates system $\left.Z_{1}, \ldots, Z_{k}, Y_{1}, \ldots, Y_{n-k}\right)$ and $E_{i}^{(0)} \in S, 1 \leq i \leq n-k$.

We show that there is a fraction of $(1-2 \gamma) /(2(1-\gamma))$ of all $T_{\alpha}$ 's that give the correct outputs for $E$ and for at least $(1-2 \gamma)^{2}(n-k)$ many among $E_{i}^{(0)}, 1 \leq i \leq n-k$. Indeed, assuming the contrary we partition all $T_{\alpha}$ 's into three (disjoint) pieces. In the first the output for $E$ is incorrect (its fraction is at most $\gamma$ ). In the second (which is desirable for our goal) the fraction of correct outputs for $E_{i}^{(0)}, 1 \leq i \leq n-k$, is at least $(1-2 \gamma)^{2}$ (its fraction is at most $(1-2 \gamma) /(2(1-\gamma))$ by the assumption). The rest of the $T_{\alpha}$ 's comprise the third piece. Thus, the total fraction of correct outputs for all $E_{i}^{(0)}, 1 \leq i \leq n-k$, together does not exceed

$$
\left(\gamma+\frac{1-2 \gamma}{2(1-\gamma)}\right)+(1-2 \gamma)^{2}\left(1-\gamma-\frac{1-2 \gamma}{2(1-\gamma)}\right)=1-2 \gamma+4 \gamma^{2}-4 \gamma^{3}<1-\gamma,
$$

that contradicts the requirement on the error probability $\gamma$.

Take such $T_{\alpha_{0}}$ and some $1 \leq i_{0} \leq n-k$ for which $T_{\alpha_{0}}$ gives the correct output. Denote by $f_{1}, \ldots, f_{t}$ the testing polynomials along the path in $T_{\alpha_{0}}$ followed by the input $E$. We claim that $Y_{i_{0}}$ occurs in one of the leading terms $\operatorname{lm}\left(f_{1, s_{1}}\right), \ldots, \operatorname{lm}\left(f_{t, s_{t}}\right)$ (thereby, $Y_{i_{0}}$ occurs in $\operatorname{lm}\left(f_{1, s_{1}} \cdots f_{t, s_{t}}\right)=\operatorname{lm}\left(f_{1, s_{1}}\right) \cdots \operatorname{lm}\left(f_{t, s_{t}}\right)$, see above $)$.

Suppose the contrary. Let $\operatorname{lm}\left(f_{l, s_{l}}\right)=\beta Z_{1}^{m_{1}^{\prime}} \cdots Z_{k}^{m_{k}^{\prime}} Y_{1}^{m_{1}} \cdots Y_{n-k}^{m_{n-k}}$, then $m_{i_{0}}=0$ for each $1 \leq l \leq t$ by the supposition. Then (1) from Section 1 implies that $\operatorname{sgn}\left(\overline{f_{l}}\left(E_{i_{0}}^{(0)}\right)\right)=$ $\operatorname{sgn}(f_{l, s_{l}}^{(n-k)} \underbrace{(0, \ldots, 0)}_{k}) \neq 0$ because of the choice of the origin of the coordinates system $Z_{1}, \ldots, Z_{k}, Y_{1}, \ldots, Y_{n-k}$. By the same token $\operatorname{sgn}\left(\overline{f_{l}}(E)\right)=\operatorname{sgn}(f_{l, s_{l}}^{(n-k)} \underbrace{(0, \ldots, 0)}_{k})$. Therefore, $E_{i_{0}}^{(0)}$ satisfies all the tests along the path under consideration in $T_{\alpha_{0}}$ followed by the input $E$, hence the output of $T_{\alpha_{0}}$ for the input $E_{i_{0}}^{(0)}$ is the same as for the input $E$, so incorrect, that contradicts the choice of $i_{0}$.

(b) First we show that $E \in S^{+}$. Take any hyperplane $H_{l}=\left\{\kappa_{1} Z_{1}+\cdots+\kappa_{k} Z_{k}+\right.$ $\left.\beta_{1} Y_{1}+\cdots+\beta_{n-k} Y_{n-k}+\beta_{0}=0\right\}, 1 \leq l \leq m$, given by linear function $L_{H_{l}}$ with the coefficients $\kappa_{i}, \beta_{j} \in \mathbb{R}$. We need to show that $L_{H_{l}}(E) \geq 0$. Let $0 \leq j_{0} \leq n-k$ be the uniquely defined index such that $\beta_{0}=\cdots=\beta_{j_{0}-1}=0, \beta_{j_{0}} \neq 0$ (if all $\beta_{0}=$ 
$\cdots=\beta_{n-k}=0$, then $L_{H_{l}}(E)=0$ ). We prove that $\beta_{j_{0}}>0$, this would entail that $\operatorname{sgn}\left(L_{H_{l}}(E)\right)=\operatorname{sgn}\left(\beta_{j_{0}}\right)>0$. Because $\operatorname{dim}\left(H_{i_{n-k}} \cap \cdots \cap H_{i_{j_{0}+1}} \cap S^{+}\right)=k+j_{0}$ and $\operatorname{dim}\left(H_{i_{n-k}} \cap \cdots \cap H_{i_{j_{0}+1}} \cap H_{i_{j_{0}}}\right)=k+j_{0}-1$ (see the beginning of this section), there exists a point $v_{n-j_{0}} \in\left(H_{i_{n-k}} \cap \cdots \cap H_{i_{j_{0}+1}} \cap S^{+}\right)-H_{i_{j_{0}}}$, notice that in the chosen coordinate system $v_{n-j_{0}}=(\underbrace{0, \ldots, 0}_{k}, y_{1}^{\left(n-j_{0}\right)}, \ldots, y_{j_{0}}^{\left(n-j_{0}\right)}, 0, \ldots, 0)$. Then $y_{j_{0}}^{\left(n-j_{0}\right)} \neq 0$, therefore $y_{j_{0}}^{\left(n-j_{0}\right)}>0$ since $v_{n-j_{0}} \in S^{+}$. Hence $0<\operatorname{sgn} L_{H_{l}}\left(v_{n-j_{0}}\right)=\operatorname{sgn}\left(\beta_{j_{0}} \cdot y_{j_{0}}^{\left(n-j_{0}\right)}\right)$, this implies that $\operatorname{sgn}\left(\beta_{j_{0}}\right)>0$. Thus $E \in S^{+}$.

Notice that the points

$$
\begin{aligned}
E_{i}^{(+)}= & (\underbrace{0, \ldots, 0}_{k}, \varepsilon_{k+1} \varepsilon_{n+1}, \ldots, \varepsilon_{k+i-1} \varepsilon_{n+1}, \\
& \left.\quad-\varepsilon_{k+i} \varepsilon_{n+1}, \varepsilon_{k+i+1} \varepsilon_{n+1}, \ldots, \varepsilon_{n} \varepsilon_{n+1}\right) \notin S^{+}, \quad 1 \leq i \leq n-k .
\end{aligned}
$$

The rest of the proof is similar to (a), but replacing the role of the points $E_{i}^{(0)}$ by $E_{i}^{(+)}$. In a similar way if $m_{i_{0}}=0$, then $\operatorname{sgn}\left(\overline{f_{l}}\left(E_{i_{0}}^{(+)}\right)\right)=\operatorname{sgn}\left(f_{l, s_{l}}^{(n-k)}(\underbrace{0, \ldots, 0}_{k})\right)=\operatorname{sgn}\left(\overline{f_{l}}(E)\right) \neq$ 0 , again because of (1) from Section 1.

Lemma 4 is proved.

An analogue of Lemma 2 from [12] and [11] is the following lemma.

Lemma 5. For any positive constants $c, c_{1}, c_{2}, c_{3}$ there exists $c_{4}>0$ satisfying the following. Let $\mathcal{S}=S$ or $\mathcal{S}=S^{+}$fulfill the conditions of the theorem. Assume that $C T T^{\prime}$ for some constant $\eta>1-c$ satisfies the inequality $\operatorname{Var}^{(\Gamma)}\left(T^{\prime}\right) \geq \eta(n-k)$ for at least $M \geq c_{3}\left(m^{c(n-k)}\right)$ of $k$-faces $\Gamma$ of $\mathcal{S}$. Then the depth $t$ of $T^{\prime}$ is greater than $c_{4}(n \log m)$.

The proof of Lemma 5 differs from the proof of the analogous Lemma 2 from [12] proved for $d$-decision trees, in which the degrees of the testing polynomials do not exceed $d$, rather than computation trees (considered in the present paper), in which the degrees of the testing polynomials could be exponential in depth $t$ of CT. It also differs from the proof of Lemma 2 of [11] where the main tool was the lower bound on the border complexity. Here the proof of Lemma 5 is much easier than in [12] and [11] and relies on the corollary (see Section 2) in which the multiplicative complexity of a polynomial is bounded from below in terms of the number of strongly singular faces of an arrangement.

Before proving Lemma 5 we show how to deduce the theorem from Lemmas 4 and 5. Consider RCT $\left\{T_{\alpha}\right\}$ recognizing $\mathcal{S}$ with error probability $\gamma<\frac{1}{2}$. Lemma 4 and counting imply the existence of $T_{\alpha_{0}}$ such that the inequality $\operatorname{Var}^{(\Gamma)}\left(T_{\alpha_{0}}\right) \geq(1-2 \gamma)^{2}(n-k)$ is true for $M=((1-2 \gamma) / 2(1-\gamma)) \Omega\left(m^{c(n-k)}\right)$ of $k$-faces $\Gamma$ of $\mathcal{S}$. Apply Lemma 5 to $\mathrm{CT} T^{\prime}=T_{\alpha_{0}}$ with $\eta=(1-2 \gamma)^{2}$. Since the error probability $\gamma$ could be made a positive constant as close to zero as desired at the expense of increasing by a constant factor the depth of RCT [21], take $\gamma$ such that $\eta>1-c$. Then Lemma 5 entails that $t \geq \Omega(n \log m)$, which proves the theorem. Thus, it remains to prove Lemma 5 .

Proof of Lemma 5. To each $k$-face $\Gamma$ of $\mathcal{S}$ satisfying the inequality $\operatorname{Var}^{(\Gamma)}\left(T^{\prime}\right) \geq$ $\eta(n-k)$, we attach a path in $T^{\prime}$ with the testing polynomials $f_{1}, \ldots, f_{t_{0}} \in \mathbb{R}\left[X_{1}, \ldots, X_{n}\right]$, 
$t_{0} \leq t$, such that $\operatorname{Var}^{(\Gamma)}\left(f_{1} \cdots f_{t_{0}}\right)=\operatorname{Var}^{(\Gamma)}\left(T^{\prime}\right)$ (in other words, $\Gamma$ is a strongly singular $k$-face for $f_{1} \cdots f_{t_{0}}$, see Section 1). Denote $f=f_{1} \cdots f_{t_{0}}$

Assume that $3^{t} \leq O\left(m^{(\eta-1+c)(n-k) / 2}\right)$, otherwise we are done. Then there exists a path of $T^{\prime}$ (we keep the notation $f_{1}, \ldots, f_{t_{0}}$ for the testing polynomials along this path) which corresponds to at least $N=\Omega\left(m^{(c-\eta+1)(n-k) / 2}\right)$ of strongly singular $k$-faces $\Gamma$ for $f$ (because there are most $3^{t}$ paths in $\left.T^{\prime}\right)$. The corollary from Section 2 implies that the multiplicative complexity $C(f) \geq \frac{1}{3}((\eta-1+c)(n-k) \log m-4 n-$ const $)$. Obviously $C(f) \leq t+t_{0}-1 \leq 2 t-1$ (see the proof of Theorem 2 of [8]). Hence $t \geq \Omega(n \log m)$ that proves Lemma 5 .

\section{Applications and Open Problems}

As a consequence of the theorem from the previous section we deduce the complexity lower bound $\Omega(n \log n)$ for any RCT, recognizing the DISTINCTNESS problem $\bigcup_{1 \leq i<j \leq n}\left\{X_{i}=X_{j}\right\} \subset \mathbb{R}^{n}$ (for estimation of the number of [n/2]-faces necessary in the theorem see [12]).

Also we get the lower bound $\Omega\left(n^{2}\right)$ for the KNAPSACK problem

$$
\bigcup_{I \subset\{1, \ldots, n\}}\left\{\sum_{i \in I} x_{i}=1\right\},
$$

this result was already obtained in [11]. It would be interesting to extend the obtained bound to other types of sets, rather than considered in the theorem polyhedra and the unions of hyperplanes. The linear $O(n)$ complexity RCT from [5] for the SET EQUALITY problem $\left\{\left(x_{1}, \ldots, x_{n}, y_{1}, \ldots, y_{n}\right):\left\{x_{1}, \ldots, x_{n}\right\}\right.$ is a permutation of $\left.\left\{y_{1}, \ldots, y_{n}\right\}\right\} \subset \mathbb{R}^{2 n}$ provides evidence that the lower bound from the theorem could not be directly extended even to such quite natural sets like the unions of planes.

Generalizing the construction of [5] we design an RCT for recognizing the following set: $\Delta_{n, m}^{(k)}=\left\{\left(x_{1}, \ldots, x_{n}, y_{1}, \ldots, y_{m}\right)\right.$ : each of both the differences of the multisets $\left\{x_{1}, \ldots, x_{n}\right\}$ and $\left\{y_{1}, \ldots, y_{m}\right\}$ contains at most $k$ elements $\} \subset \mathbb{R}^{n+m}$. Evidently, $k \geq$ $|n-m|$. Denote the polynomials $f(X)=\left(X-x_{1}\right) \cdots\left(X-x_{n}\right)$ and $g(X)=(X-$ $\left.y_{1}\right) \cdots\left(X-y_{m}\right)$. First compute (deterministically) $f\left(z_{i}\right), g\left(z_{i}\right)$ at $2 k+1$ random points, $0 \leq i \leq 2 k$, with the complexity $O(k(n+m))$. Then (deterministically) interpolate the rational function $h=f / g$, being (presumably) a quotient of two monic polynomials both of degrees at most $k$ by means of its values $(f / g)\left(z_{i}\right), 1 \leq i \leq 2 k$, with the complexity $O\left(k \log ^{2} k\right)$ [3]. Finally, (deterministically) check whether the value of the rational function $h\left(z_{0}\right)$ obtained coincides with $f\left(z_{0}\right) / g\left(z_{0}\right)$. The complexity $O(k(n+$ $m)$ ) of the designed RCT is better than the complexity $O((n+m) \log (n+m))$ of an obvious CT based on a sorting algorithm when $k$ is small enough.

\section{Acknowledgment}

I would like to thank Marek Karpinski for useful discussions. 


\section{References}

1. W. Baur, V. Strassen, The complexity of partial derivatives, Theoret. Comput. Sci., Vol. 22, 1983, pp. 317330 .

2. M. Ben-Or, Lower bounds for algebraic computation trees, Proc. ACM Symp. Theory Comput., 1983, pp. $80-86$

3. D. Bini, V. Pan, Polynomial and Matrix Computations, Birkhäuser, Basel, 1994.

4. A. Bjorner, L. Lovasz, A. Yao, Linear decision trees: volume estimates and topological bounds, Proc. ACM Symp. Theory Comput., 1992, pp. 170-177.

5. P. Buergisser, M. Karpinski, T. Lickteig, On randomized algebraic test complexity, J. Complexity, Vol. 9, 1993, pp. 231-251.

6. A. Chistov, D. Grigoriev, Solving systems of algebraic equations in subexponential time I, II, Preprints LOMI E-9-83, E-10-83, Leningrad, 1983.

7. D. Grigoriev, Nearly sharp complexity bounds for multiprocessor algebraic computations, J. Complexity, Vol. 13, No. 1, 1997, pp. 50-64.

8. D. Grigoriev, Complexity lower bounds for randomized computation trees over algebraically closed fields, submitted to Comput. Complexity.

9. D. Grigoriev, M. Karpinski, Lower Bounds on Complexity of Testing Membership to a Polygon for Algebraic and Randomized Computation Trees, Technical Report TR-93-042, International Computer Science Institute, Berkeley, CA, 1993.

10. D. Grigoriev, M. Karpinski, Lower Bound for Randomized Linear Decision Tree Recognizing a Union of Hyperplanes in a Generic Position, Research Report No. 85114-CS, University of Bonn, 1994.

11. D. Grigoriev, M. Karpinski, Randomized quadratic lower bound for knapsack, Proc. ACM Symp. Theory Comput., 1997, pp. 76-85.

12. D. Grigoriev, M. Karpinski, F. Meyer auf der Heide, R. Smolensky, A lower bound for randomized algebraic decision trees, Proc. ACM Symp. Theory Comput., 1996, pp. 612-619.

13. D. Grigoriev, M. Karpinski, R. Smolensky, Randomization and the computational power of analytic and algebraic decision trees, Comput. Complexity, Vol. 6, No. 4, 1997, pp. 376-388.

14. D. Grigoriev, M. Karpinski, N. Vorobjov, Improved lower bound on testing membership to a polyhedron by algebraic decision trees, Proc. 36th IEEE FOCS, 1995, pp. 258-265.

15. D. Grigoriev, M. Karpinski, N. Vorobjov, Lower bound on testing membership to a polyhedron by algebraic decision and computation trees, Discrete Comput. Geom., Vol. 17, No. 2, 1997, pp. 191-215.

16. D. Grigoriev, N. Vorobjov, Solving systems of polynomial inequalities in subexponential time, J. Symbolic Comput., Vol. 5, 1988, pp. 37-64.

17. J. Heintz, Definability and fast quantifier elimination in algebraically closed fields, Theoret. Comput. Sci., Vol. 24, 1983, pp. 239-277.

18. S. Lang, Algebra, Addison-Wesley, New York, 1965.

19. T. Lickteig, On semialgebraic decision complexity, Preprint TR-0-052 ICSI, Berkeley, CA, 1990.

20. U. Manber, M. Tompa, Probabilistic, nondetemrinistic and alternating decision trees, Proc. 14th ACM STOC, 1982, pp. 234-244.

21. F. Meyer auf der Heide, Simulating probabilistic by deterministic algebraic computation trees, Theoret. Comput. Sci., Vol. 41, 1985, pp. 325-330.

22. J. Montana, J. Morais, L. Pardo, Lower bounds for arithmetic network, II: sum of Betti numbers, Appl. Algebra Engrg. Comm. Comput., Vol. 7, 1996, pp. 41-51.

23. J. Montana, L. Pardo, Lower bounds for arithmetic networks, Appl. Algebra Engrg. Comm. Comput., Vol. 4, 1993, pp. 1-24.

24. D. Mumford, Algebraic Geometry, Springer-Verlag, New York, 1976.

25. I. R. Shafarevich, Basic Algebraic Geometry, Vol. 1, Springer-Verlag, New York, 1994.

26. M. Steele, A. Yao, Lower bounds for algebraic decision trees, J. Algorithms, Vol. 3, 1982, pp. 1-8.

27. V. Strassen, Die Berechnungskomplexitaet von elementarsymmetrischen Funktionen und von Interpolationskoeffizienten, Numer. Math., Vol. 20, 1973, pp. 238-251.

28. V. Strassen, Computational complexity over finite fields, SIAM J. Comput., Vol. 5, No. 2, 1976, pp. 324331 . 
29. A. Tarski, A Decision Method for Elementary Algebra and Geometry, University of California Press, Berkeley, CA, 1951.

30. A. Yao, Algebraic decision trees and Euler characteristic, Proc. IEEE Symp. Found. Comput. Sci., 1992, pp. 268-277.

31. A. Yao, Decision tree complexity and Betti numbers, Proc. ACM Symp. Theory Comput., 1994, pp. 615624.

Received May 14, 1997, and in revised form October 27, 1997, and February 16, 1998. 